\title{
Neuroprotective effect of melatonin on nickel-induced affective and cognitive disorders and oxidative damage in rats
}

\author{
Mouloud Lamtai1 $^{1}$, Sihame Ouakki ${ }^{1}$, Oussama Zghari1 ${ }^{1}$, Abdelghafour El Hamzaoui ${ }^{1}$, Hajar Benmhammed ${ }^{1}$, Sofia \\ Azirar1 $^{(\mathbb{D}, \text { Aboubaker El Hessni1 }}{ }^{1}$, Abdelhalem Mesfioui ${ }^{1}$, Ali Ouichou ${ }^{1}$. \\ ${ }^{1}$ Laboratory of Genetics, Neuroendocrinology and Biotechnology, Faculty of Science, University Ibn Tofail, 14000, Kenitra, \\ Morocco. \\ *Correspondence: mouloud.lamtai@uit.ac.ma
}

Received: September 18, 2020 Accepted: November 18, 2020

\begin{abstract}
The present work is carried out to explore the neuroprotective potential of Melatonin(Mel), on Ni-induced neurobehavioral, biochemical and histological alterations in male and female rats. The rats were intraperitoneally administered by nickel chloride $\left(\mathrm{NiCl}_{2}, 1 \mathrm{mg} / \mathrm{kg}\right)$ and $\mathrm{Mel}(4 \mathrm{mg} / \mathrm{kg})$ for 60 days. A neurobehavioral assessment was performed. Biochemical determinations of oxidative stress (OS) levels, and histological analysis of hippocampal tissues were also performed. Results showed that Nickel (Ni) treatment increased anxiety-like and depression-like behavior in rats. Besides, cognitive behavior on the Morris water maze was compromised following Ni treatment. Alongside this, $\mathrm{Ni}$ elevated hippocampal OS markers like lipid peroxidation and nitric oxide formation with a decrease in superoxide dismutase and catalase activities. Histological observations confirmed these results. Significantly, Mel administration alleviated neurobehavioral changes in Ni-treated rats of both genders. Also, Mel attenuated Ni-induced OS and increased the activities of antioxidant enzymes. The histopathological studies in the hippocampus supported that Mel markedly reduced the $\mathrm{Ni}$-induced neuronal loss. In conclusion, this study suggests that Mel has a neuroprotective effect against Ni-induced neurobehavioral alterations, which may be related to lowering OS in the hippocampus.
\end{abstract}

Keywords: Nickel, Melatonin, Depression-like, Anxiety-like, Memory, Oxidative stress

\section{Introduction}

Nickel (Ni) is a ubiquitous metal in the environment [1] and is frequently used in various consumer and industrial applications due to its unique physical and chemical properties [2]. At the same time, Ni is known to be an essential element for several animal species [3]. Its deficiency can adversely affect different body functions [4].

On the other hand, environmental and occupational exposure to $\mathrm{Ni}$ at high levels can cause various adverse effects on human health [1]. Generally, humans may be exposed to Ni through food, water, and air, as it is produced in mining, extraction, refining, electroplating, food processing, and $\mathrm{Ni}$ waste disposal [2]. Occupational exposure has been shown to give rise to elevated levels of $\mathrm{Ni}$ in blood, urine, and body tissues [5]. This metal overload was found to have teratogenic, reproductive toxic, pulmonary toxic, nephrotoxic, and hepatotoxic effects in living organisms [1, 6]. Based on Ni workers and laboratory animals' studies, all Ni compounds have been classified as a human carcinogen by the International Agency for Research on Cancer [1, 7]. However, studies on the neurotoxic effects of Ni are sparse given the environmental health problem associated with Ni. Neurological effects such as giddiness and weariness were reported in electroplating plant workers who drank water contaminated with nickel chloride $\left(\mathrm{NiCl}_{2}\right)\left(1.63 \mathrm{~g} / \mathrm{dm}^{3}\right)$ [5]. The same effects were observed in an evaluation of workers welding high-Ni alloys, exposed for six weeks to Ni fumes $\left(0.07\right.$ to $\left.1.1 \mathrm{mg} \mathrm{Ni} / \mathrm{m}^{3}\right)$ [8]. In animal studies, Ni exposure induced aggressive behavior, emotional changes, and cognitive problems [9, 10].

The mechanism by which Ni induces neurological damage is partially known. Generally, a large body of research showed a potential link between oxidative stress (OS) and the etiology of affective and cognitive dysfunctions [11, 12]. In this sense, one of the possible mechanisms behind Ni neurotoxicity has been attributed to OS [1]. Ni is known to disrupt the pro-/antioxidant balance in the cells [13], which may precede neurobehavioral disorders. Our previous study showed that chronic Ni exposure induced depression-like, anxiety-like, memory deficit, and alterations in OS markers in the hippocampus [13], a crucial brain region in learning, memory processes, and emotion regulation [14, 15]. These effects are similar to those induced by other heavy metals such as Copper $(\mathrm{Cu})$, Cadmium $(\mathrm{Cd})$ and Aluminum (Al) [16-19]. Certain 
regions of the central nervous system (CNS), such as the hippocampus, may be particularly sensitive to OS [20]. Therefore, molecules that inhibit OS potentially protect neuronal cells and subsequently prevent neurobehavioral disturbances.

Melatonin (Mel) is a powerful antioxidant that is mainly synthesized and secreted by the pineal gland [21]. This neurohormone acts as a potent free radical scavenger, and it activates the major antioxidant enzymes such as superoxide dismutase (SOD) and catalase (CAT) in the brain [22]. In addition to its antioxidant properties, Mel has been studied due to its neuroprotective actions in several neurodegenerative diseases such as Alzheimer's and Parkinson's diseases [21]. Furthermore, Mel exerted anxiolytic and antidepressant effects [23, 24], and it helped to improve cognition ability [25]. To our knowledge, no investigation has reported the efficacy of $\mathrm{Mel}$ to attenuate Ni-induced neurotoxicity in adult rats. Given the foregoing, our study aimed to explore the efficacy of Mel administration in alleviating behavior, OS, and histopathological changes in the hippocampus induced by chronic exposure to Ni in male and female rats.

\section{Materials and Methods Chemicals}

$\mathrm{NiCl}_{2}$, trichloroacetic acid (TCA), thiobarbituric acid (TBA), nitroblue tetrazolium (NBT), Griess reagent, methionine, riboflavin, Triton X-100, and xylene were purchased from Sigma-Aldrich Chemical CO (St. Louis, MO, USA). Melatonin (N-acetyl-5-methoxytryptamine) was purchased from Sigma-Aldrich (St. Louis, France). It was dissolved just before use in a vehicle composed of $1 \%$ ethanol and $99 \%$ distilled water. Hydrogen peroxide $\left(\mathrm{H}_{2} \mathrm{O}_{2}\right)$, physiological saline buffer $(0.9 \% \mathrm{NaCl})$, ethanol $(70 \%)$, chloral hydrate, paraformaldehyde, and Butanol used in this work were purchased from a standard commercial supplier (OUAMEB, Kenitra, Morocco). All other chemicals were of the highest analytical grade.

\section{Animals}

Adult Wistar rats of both genders (approximately 60 days old and with mean weight gain of $120 \pm 20$ g), born and raised in Animal House in Ibn Tofail University, were used in this study. The adult rodents were maintained under a 12-h light/dark cycle at a temperature of $22 \pm 1^{\circ} \mathrm{C}$ with free access to water and food. All experiments were performed according to the National Institutes of Health Guide for the Care and Use of Laboratory Animals. All efforts were made to minimize the number of rats and their suffering.

\section{Experimental design and Treatments}

A total of forty eight rats were divided into 4 groups for each gender:

(1) Control $\left(n=6 \%\right.$ and $\left.60^{7}\right)$ : Physiological saline buffer $(0.9 \% \mathrm{NaCl})$ was given;

(2) $\mathrm{Mel}\left(n=6 \%\right.$ and $\left.60^{\top}\right)$ : Only melatonin at a dose of $4 \mathrm{mg} / \mathrm{kg}$ body weight was given [13];

(3) $\mathrm{Ni}\left(n=6 \%\right.$ and $\left.60^{\prime}\right)$ : Nickel chloride was given at a dose of $1 \mathrm{mg} / \mathrm{kg}$ body weight [24, 26];

(4) $\mathrm{Ni}+\mathrm{Mel}\left(n=6 \%\right.$ and $\left.60^{\top}\right)$ : Melatonin at a dose of $4 \mathrm{mg} / \mathrm{kg}$ body weight together with nickel chloride at a dose of $1 \mathrm{mg} / \mathrm{kg}$ body weight was given

For each group, the treatment was given intraperitoneally for two months before the behavioral parameter estimation was done. Following neurobehavioural studies, all the rats were sacrificed and the hippocampi were rapidly excised and processed for subsequent histological and biochemical analysis.

\section{Neurobehavioral tests \\ Anxiety-Like Measurement}

The open field test (OFT) [27] and the elevated plus maze (EPM) [28] were used to measure the anxietylike behavior in rodents. The protocol of each test is well detailed in our previous work [13]

\section{Depression-Like Measurement in Forced Swimming Test (FST)}

The Forced Swimming Test (FST) is the most widely used pharmacological model for evaluating depressive-like responses in rodents [29]. The test involved putting the rat into an inescapable cylinder (diameter $10 \mathrm{~cm}$, height $25 \mathrm{~cm}$ ) of water at $23-25^{\circ} \mathrm{C}$ for a period of $5 \mathrm{~min}$. The total amount of time each animal remained immobile was recorded as immobility time (TIM). An increase in the TIM is an indicative of depression-like behavior.

\section{Cognitive Measurement}

Morris Water Maze Test (MWM)

Spatial learning and memory performances were assessed by monitoring behavior in the Morris Water Maze Test (MWM) task as described by Morris (1984) [30]. The apparatus was made of circle polypropylene pool (110 cm in diameter and $50 \mathrm{~cm}$ in height) filled with white-opaque water $\left(24 \pm 1^{\circ} \mathrm{C}\right)$. The test is predicated on the rodent finding the invisible platform (submerged $1 \mathrm{~cm}$ below the water surface) using numerous extra maze cues. During the acquisition phase (the 
first 4 days), the escape latency (s) of each rat to reach the hidden platform was recorded. In the probe test phase (the 5th day), the invisible platform was removed from pool and each rat received a free swimming of 60 s' duration. The percent time spent in the target quadrant was measured.

\section{Estimation of oxidative stress parameters}

The neurochemical analysis was done to assess concentrations of thiobarbituric acid reactive substances ( TBARS) content, nitric oxide (NO), superoxide dismutase (SOD) and catalase (CAT) activities in the hippocampus o $\mathrm{f}$ rats. The hippocampi were collected and rinsed in phosphate-buffered saline $(50 \mathrm{mM} \mathrm{W} / \mathrm{V}, \mathrm{pH}: 7.4)$, then homoge nized using a homogenizer and centrifuged at $1500 \mathrm{rpm}$ for $10 \mathrm{~min}$ at $4{ }^{\circ} \mathrm{C}$ [58]. The resulting supernatants were $\mathrm{f}$ rozen at $-20{ }^{\circ} \mathrm{C}$ until further use.

Hippocampal LPO was estimated according to the procedure of Draper and Hadley [32]. Hippocampal NO was determined using Griess reagent [33]. The SOD and CAT activities were analyzed using the protocol of Beauchamp and Fridovich [34] and the protocol of Aebi [35], respectively.

a

\section{Behavioral Assessment}

Adult Wistar rat

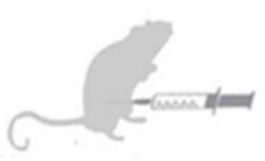

60 days

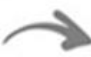

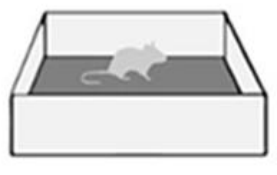

OFT

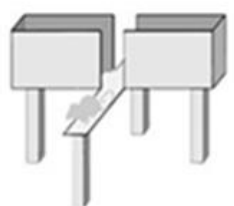

EPM

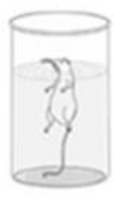

FST

\footnotetext{
- Control group $(n=6 \odot$ and $6 \hat{\jmath}): 0.9 \% \mathrm{NaCl}$.

- Mel group $(n=6$ + and $6 \hat{\jmath}): 4 \mathrm{mg} / \mathrm{kg} /$ day.

- $\operatorname{Nigroup}(n=6$ ㅇ and $6 \hat{\jmath}): 1 \mathrm{mg} / \mathrm{kg} /$ day.

- $\mathrm{Ni}+\operatorname{Mel} \operatorname{group}(n=6$ 古 and $6 \hat{\jmath})$ :

$\mathrm{Ni}(1 \mathrm{mg} / \mathrm{kg} /$ day $)+\mathrm{Ni}(4 \mathrm{mg} / \mathrm{kg} /$ day .
}

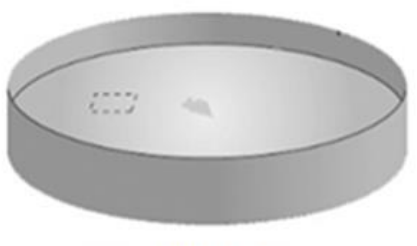

MWM

\section{b Biochemical analysis}

- LPO

- NO

- SOD

- CAT
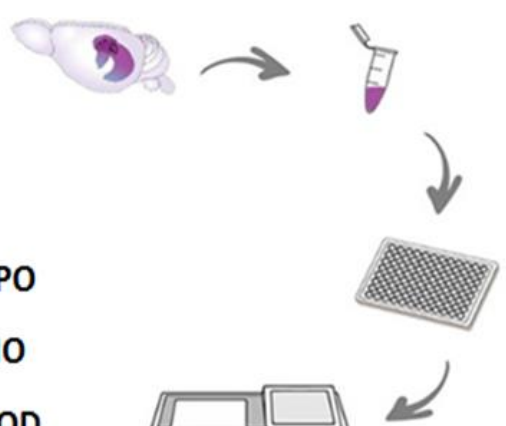

C

Histological analysis

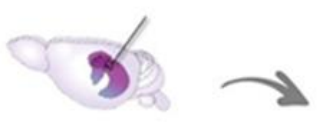

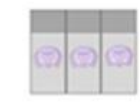
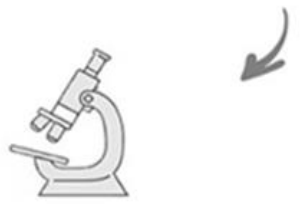

Figure 1. Sample description and experimental steps of nickel (Ni) and melatonin (Mel) administration protocol. (a). After 60 days, the behavioral test assessment through Open Field test (OFT), Elevated Plus Maze (EPM), Forced Swimming Test (FST) and Morris Water Maze (MWM) Test were performed. The animals were euthanized, Thereafter, (b) oxidative stress markers (lipid peroxidation (LPO), nitric oxide (NO) levels, superoxide dismutase (SOD) and catalase (CAT)) activities) and (c) histology of the hippocampus was evaluated. 


\section{Histological analysis}

For histological studies, brains were collected in $4 \%$ paraformaldehyde for proper fixation. Sections of the hippocampus with $30 \mu \mathrm{m}$ of thickness were made and stained with cresyl violet for examination [36].

The experimental design of all methodological steps is summarized in Figure. 1.

\section{Statistical analysis}

All analyses were carried out in SPSS (version 22). Comparisons among groups were performed using on e-way analysis of variance (ANOVA). The escape latency in the MWM test navigation phase was analyzed by twoway analysis of variance (ANOVA) with repeated measures. All data are reported as mean \pm standard error of the mean (SEM) and probability values (p) of less than 0.05 were considered to be statistically significant.

\section{Results}

\section{Effect of Nickel and Melatonin on the Levels of Anxiety-Like Measured in the Open Field test (OFT)}

The data recorded for the levels of anxiety of rats are shown in Table 1. Ni exposure significantly decreased the TCA in male $(-70 \%)(p<0.001)$ and female $(-44 \%)(p<0.01)$ rats when compared to their controls. Also, the NRC was significantly decreased in Ni treated male rats $(-76 \%)(p<0.001)$ and female rats $(-34 \%)(p<0.05)$ when compared to control rats. Contrarily, Mel administration significantly increased the TCA $(\mathrm{p}<0.05)$ and the NRC, especially in females $(\mathrm{p}<0.05)$, which are diminished in the Ni treated groups (Table 1). No significant difference is observed for NTS on the OFT ( $p>0.05)$

\section{Effect of Nickel and Melatonin on Anxiety Levels Measured in Elevated Plus Maze Test (EPM)}

As shown in Table 2, the TOA was significantly decreased in Ni treated male rats by $67 \%(\mathrm{p}<0.001)$ and female rats by $47 \%(p<0.01)$ when compared to control rats, suggesting that anxious behavior was increased following Ni exposure. However, following Mel supplementation TOA was significantly $(\mathrm{p}<0.05)$ increased in female rats $(+66 \%)$, whereas, in males, a non-significant increase in TOA was observed $(+45 \%)$ compared to Ni treated rats $(\mathrm{p}>0.05)$. Mel is more effective in female rats compared to male rats $(\mathrm{p}<0,001)$. In contrast, $\mathrm{Ni}$ and/or Mel had no significant effect on EOA and locomotor activity represented by the TEA $(\mathrm{p}>0.05)$.

Table 1. Effect of melatonin $(4 \mathrm{mg} / \mathrm{kg})$ on anxiety-related behavior of male and female Nickel $(\mathrm{Ni})(1 \mathrm{mg} / \mathrm{kg})$ exposed rats subjected to the open field test. TCA: the time spent in the center of the area; NRC: the number of returns to the center; NTS: the number of total squares crossed.

\begin{tabular}{clll}
\hline Group & TCA (Seconds) & NRC & NTS \\
\hline Males & & & \\
Control & $40.40 \pm 05.09$ & $14.50 \pm 01.59$ & $63.80 \pm 06.72$ \\
Mel & $34.60 \pm 02.62^{\# \#}$ & $13.60 \pm 01.21^{\# \# \#}$ & $65.80 \pm 08.97$ \\
$\mathrm{Ni}$ & $12.00 \pm 01.41^{* * *}$ & $03.40 \pm 00.75^{* * *}$ & $68.20 \pm 05.55$ \\
$\mathrm{Ni}+$ Mel & $22.80 \pm 00.73^{* *}$ & $07.60 \pm 00.40$ & $72.00 \pm 10.49$ \\
Females & & & \\
Control & $29.80 \pm 03.90^{\# \#}$ & $10.00 \pm 00.85$ & $69.60 \pm 11.29$ \\
Mel & $36.60 \pm 02.11^{\# \#}$ & $15.25 \pm 01.16^{* *} / \# \#$ & $76.40 \pm 10.98$ \\
Ni & $16.80 \pm 01.11^{* *}$ & $06.60 \pm 00.68^{*}$ & $83.00 \pm 01.18$ \\
Ni + Mel & $26.40 \pm 01.21^{\#}$ & $09.80 \pm 01.56$ & $66.20 \pm 09.73$ \\
\hline
\end{tabular}

Data are presented as mean \pm S.E.M. ${ }^{*} \mathrm{P}<0.05$ vs. control group; and $\# \mathrm{P}<0.05$ vs. Ni treated group. 
Table 2. Effect of melatonin $(4 \mathrm{mg} / \mathrm{kg})$ on anxiety-related behavior of male and female Nickel (Ni) $(1 \mathrm{mg} / \mathrm{kg})$ exposed rats subjected to the elevated plus maze. TOA: the time spent on the open arms; EOA: the entries into open arms; TEA: The number of full entries into the arms.

\begin{tabular}{cccc}
\hline Group & TOA (Seconds) & EOA & TEA \\
\hline Males & & & $04.40 \pm 01.24$ \\
Control & $73.40 \pm 05.06$ & $03.40 \pm 00.74$ & $06.44 \pm 00.39$ \\
Mel & $63.60 \pm 04.44^{\# \#}$ & $03.20 \pm 00.37$ & $03.20 \pm 00.37$ \\
$\mathrm{Ni}$ & $24.60 \pm 02.86^{* * *}$ & $02.00 \pm 00.31$ & $05.00 \pm 00.63$ \\
$\mathrm{Ni}+\mathrm{Mel}$ & $35.75 \pm 03.40^{* * *}$ & $02.20 \pm 00.38$ & $06.40 \pm 01.07$ \\
Females & & & $06.60 \pm 00.98$ \\
Control & $75.00 \pm 07.33$ & $04.84 \pm 00.39$ & $06.00 \pm 01.14$ \\
Mel & $116.60 \pm 07.01^{* *} / \# \#$ & $06.00 \pm 00.54$ \# & $05.80 \pm 00.37$ \\
Ni & $39.80 \pm 04.44^{* *}$ & $03.40 \pm 00.51$ & $04.40 \pm 00.60$ \\
Ni + Mel & $66.40 \pm 04.20^{\#}$ &
\end{tabular}

Data are presented as mean \pm S.E.M. ${ }^{*} \mathrm{P}<0.05$ vs. control group; and $\# \mathrm{P}<0.05$ vs. Ni treated group.

Table 3. Effect of melatonin $(4 \mathrm{mg} / \mathrm{kg}$ ) on depression-related behavior of male and female $\mathrm{Ni}(1 \mathrm{mg} / \mathrm{kg})$-exposed rats subjected to the forced swimming test. TIM: immobility time; TST: struggling time.

\begin{tabular}{ccc}
\hline Group & TIM (Seconds) & TST (Seconds) \\
\hline Males & $10.00 \pm 00.70$ & $168.00 \pm 05.41$ \\
Control & $10.80 \pm 01.13^{\# \#}$ & $148.20 \pm 09.82^{\#}$ \\
Mel & $28.20 \pm 03.62^{* *}$ & $107.06 \pm 04.50^{* * *}$ \\
$\mathrm{Ni}$ & $23.40 \pm 02.21$ & $130.60 \pm 03.22$ \\
$\mathrm{Ni}+\mathrm{Mel}$ & $12.02 \pm 01.71$ & \\
Females & $05.60 \pm 00.39^{* / \# \#}$ & $171.20 \pm 02.15^{* * / \# \#}$ \\
Control & $24.40 \pm 03.19^{* *}$ & $108.80 \pm 05.25^{* * *}$ \\
Mel & $15.80 \pm 01.56^{\#}$ & $125.20 \pm 01.39^{* * / \#}$ \\
Ni &
\end{tabular}

Data are presented as mean \pm S.E.M; ${ }^{*} \mathrm{P}<0.05$ vs. control group; and $\# \mathrm{P}<0.05$ vs. Ni treated group.

Effects of Nickel and Melatonin on Depressive-Like Performances Measured by Forced Swimming Test (FST)

In the FST (Table 3), Ni treatment in both genders provoked a significant increase in immobility time (TIM) by $182 \%$ in male and by $100 \%$ in female rats when compared to saline-treated animals $(p<0.01)$, and this effect was attenuated by Mel administration especially in females in comparison with Ni-treated group ( $<<0.05)$ (Table 3). Mel is more effective in female rats compared to male rats $(\mathrm{p}<0.05)$. On the other hand, Table 3 also shows that Ni decreased significantly the struggling time (TST) in male $(-36 \%)$ and female rats $(-22 \%)$ when compared to saline-treated rats $(\mathrm{p}<0.01)$, and Mel also was able to prevent this depressogenic effect induced by $\mathrm{Ni}$, especially in female rats $(\mathrm{p}<0.05)$.

\section{Nickel and Melatonin Effects on Learning and Memory Measured by Morris Water Maze}

The mean escape latency increased throughout the learning trials in Ni groups compared to control rats, especially in males $(p<0.05)$. However, administration of the Mel non-significantly decreased this effect in Ni intoxicated rats $(p>0.05)$ (Figures 2a).

Measurements in the probe test demonstrated that the mean \% of time spent in the correct quadrant in group $\mathrm{Ni}$ dramatically exceeded the respective values in control groups of both genders $(+45 \%$ in males, $\mathrm{p}<0.05$ and $+19 \%$ in females, $\mathrm{p}>0.05)$. However, Mel and Ni treatment groups showed a non-significantly reduced \% of time spent in the correct quadrant compared with $\mathrm{Ni}$ treated groups, especially in females $(p>0.05)$ (Figure $2 b$ ). 

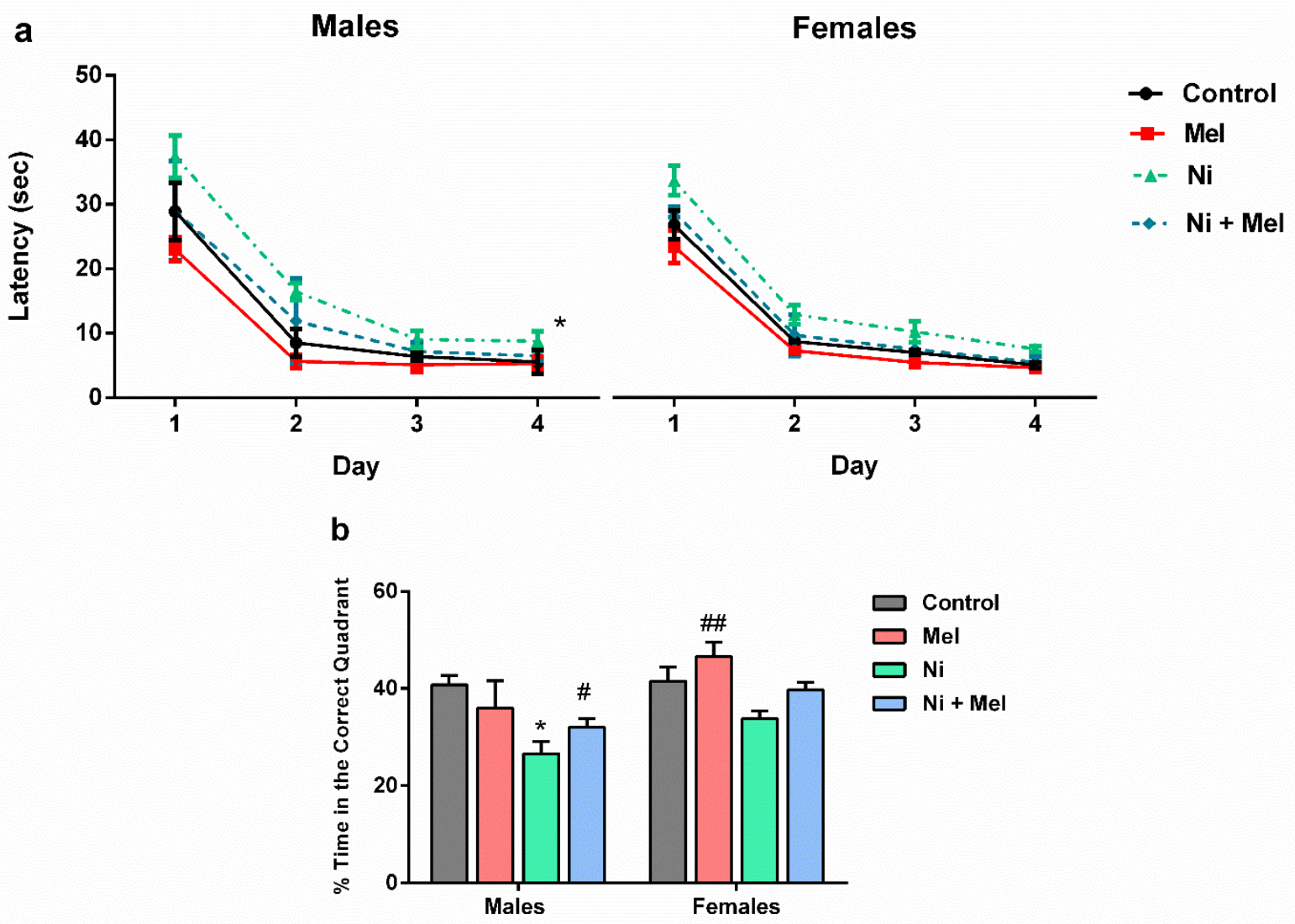

Figure 2. Effects of melatonin $(4 \mathrm{mg} / \mathrm{kg}$ ) on escape latency during the acquisition phase in the Morris Water Maze Test (MWM), in male and female (a) Nickel (Ni) $(1 \mathrm{mg} / \mathrm{kg}$ )-exposed rats. The percentage of time spent in the target quadrant (b) was measured to determine the effects on the spatial memory ability of the rat. The data obtained were expressed with mean \pm S.E.M. The significance levels are ${ }^{*} \mathrm{P}<0.05$ vs. control group; and $\# \mathrm{P}<0.05$ vs. Ni treated group.

\section{Effects of Nickel and melatonin on oxidative stress parameters in the hippocampus Lipid peroxidation product (TBARS levels)}

As observed in Figure 3a, between groups comparisons indicated Ni exposure rats significantly increased hippocampus LPO (TBARS) levels ( $\mathrm{p}<0.001$ ) by $216 \%$ and $158 \%$ in male and female Ni-treated rats when compared to saline-treated rats, respectively. After two months of Mel supplementation, TBARS levels were significantly decreased by $27 \%(p<0.01)$ and $43 \%(p<0.001)$ in male and female Ni-Mel treated rats, respectively, compared to Ni treated group. Mel is more effective in female rats compared to male rats $(\mathrm{p}<0.01)$.

\section{NO Concentrations in Hippocampus}

The intake of Ni for 8 weeks, significantly elevated NO levels in the hippocampus in male $(+96, \mathrm{p}<0.05)$ and female rats $(+64 \%, \mathrm{p}<0.05)$, as compared to the adult saline-treated rats. Moreover, Mel treatment caused a significant decrease in the hippocampus NO levels in female Ni-treated rats when compared to the Ni exposure group $(\mathrm{P}<0.05)($ Figure $3 b)$. The effect of sex was noted following Mel administration; this molecule is more effective in females than males ( $p<$ 0.05).

\section{Estimation of antioxidative enzymes activities}

As indicated in Figures 3c and 3d, SOD and CAT activities were significantly reduced upon the Ni intake, by $31 \%$ $(\mathrm{p}<0.01)$ and $36 \%(\mathrm{p}<0.001)$ in the male rats, and by $22 \%(\mathrm{p}<0.05)$ and $28 \%(\mathrm{p}<0.01)$ in the female rats, respectively, as compared to the saline-treated groups. However, Ni intoxicated rats treated with Mel showed a significant $(p<0.05)$ increase in the activities of antioxidant enzymes in the hippocampus when compared to Ni intoxicated rats. 
a

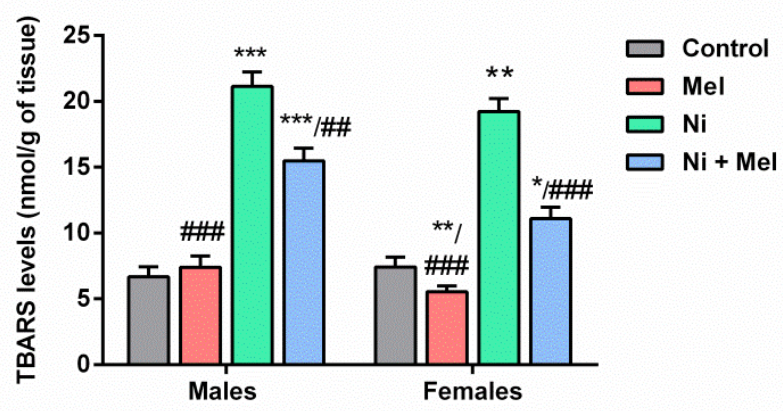

b

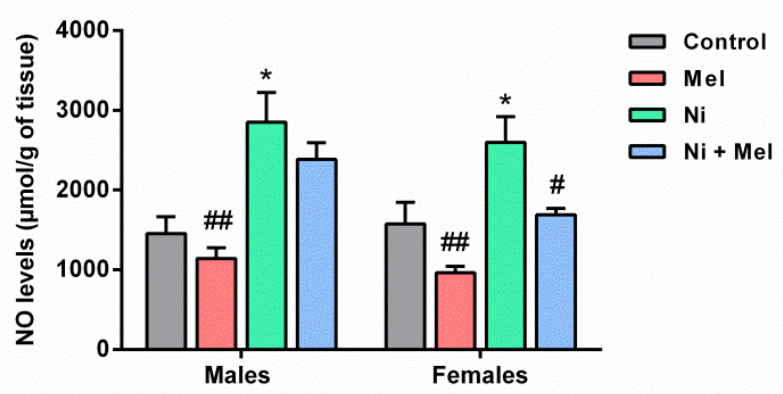

C

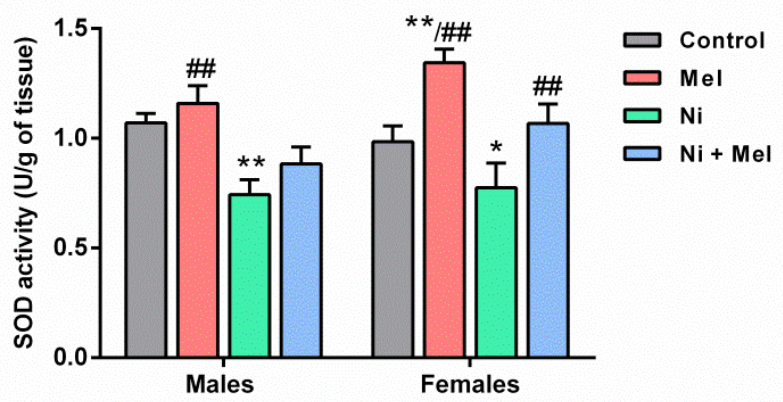

d

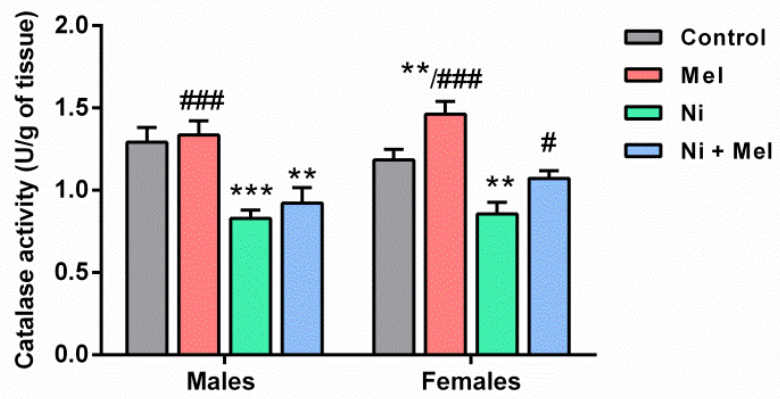

Figure 3. Effect of melatonin (4 mg/kg) on levels of (a) thiobarbituric acid reactive substances (TBARS) and (b) nitric oxide (NO), (c) superoxide dismutase (SOD) and (d) catalase (CAT) activities in the hippocampus of male and female Nickel (Ni) $(1 \mathrm{mg} / \mathrm{kg})$-exposed rats. The results obtained were expressed with \pm S.E.M. ${ }^{*} \mathrm{P}<0.05$ vs. control group; and $\# \mathrm{P}<0.05$ vs. Ni treated group.

\section{Histological analysis}

As shown in Figure 4, the mean number of intact pyramidal neurons in the CA3 region of the hippocampus was significantly decreased by $46 \%$ in males and by $31 \%$ in females Ni-treated rats, compared to control groups ( $p<0.01)$. Mel administration alone had no significant effect on neuronal survival in this layer compared to the control groups, but, following Mel treatment, the neuronal loss significantly decreased in the $\mathrm{Ni}+$ Mel groups of both genders $(\mathrm{p}<0.05$ in males and $\mathrm{p}<0.01$ in females) compared to the Ni groups.

As illustrated by Figure 5, histopathological analyses of CA3 pyramidal neurons of the control groups showed a normal histological appearance, whereas a neuronal loss, cellular disorganization, and shrinkage were observed in $\mathrm{Ni}$ treated groups of both genders compared to their normal controls. These changes were attenuated in $\mathrm{Ni}+\mathrm{Mel}$ groups compared to the Ni treated groups, showing normal pyramidal neurons (Figures 4 and 5). 


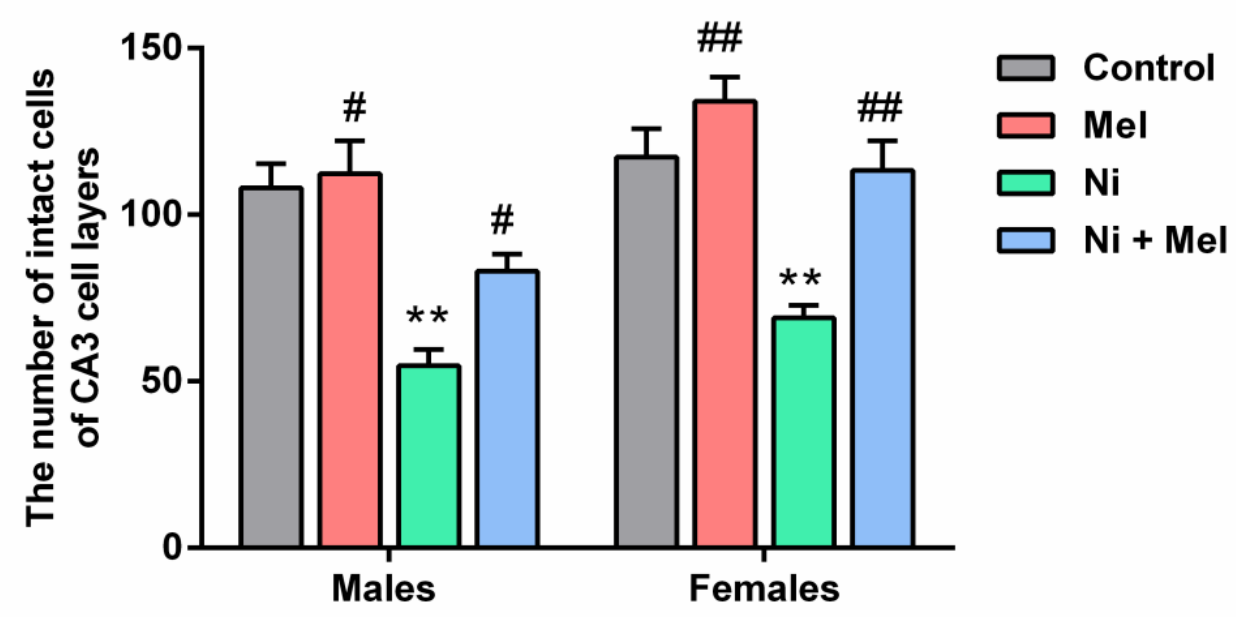

Figure 4. Effect of Melatonin (Mel) $\left(4 \mathrm{mg} / \mathrm{kg}\right.$ ) on the number of neurons in $0.18 \mathrm{~mm}^{2}$ area of CA3 region of hippocampus in male and female Nickel $(\mathrm{Ni})(1 \mathrm{mg} / \mathrm{kg})$-exposed rats. The data obtained were expressed with \pm S.E.M. ${ }^{*} \mathrm{P}<0.05$ vs. control group; and $\# \mathrm{P}<0.05$ vs. Ni treated group

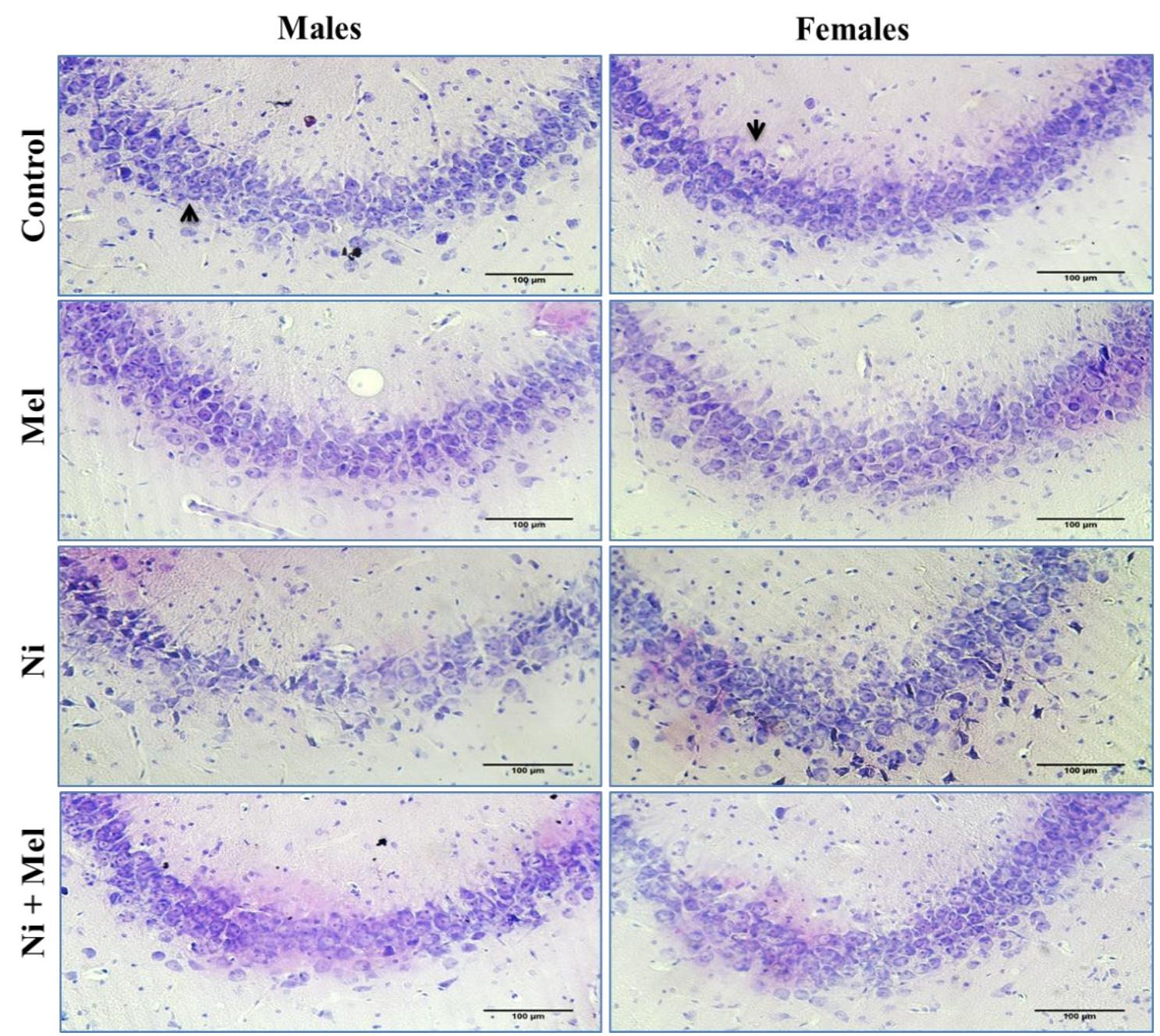

Figure 5. Light micrographs of hippocampus with cresyl violet staining in the control, Melatonin (Mel), Nickel (Ni) and $\mathrm{Ni}+\mathrm{Mel}$ groups in CA3 cell layer of hippocampus in male and female rats. Black arrows indicate the intact neurons. Bar represents $100 \mu \mathrm{m}$. 


\section{Discussion}

In the present study, $\mathrm{Ni}$ at $1 \mathrm{mg} / \mathrm{kg}$ increased anxiety-like behavior in rats of both genders. Our finding corroborates with data recently published by our research group in which $\mathrm{Ni}(0.25,0.5 \mathrm{or} 1 \mathrm{mg} / \mathrm{kg})$ caused higher anxiety in rats [13]. Our data also showed that $1 \mathrm{mg} / \mathrm{kg}$ of Ni produced higher depression-like behavior in male and female rats. This result was in accordance with the study of Kahloula et al, reporting that depression-like behavior was significantly increased by $\mathrm{Ni}$ treatment during the development period [10]. Interestingly, Mel administration (4 mg/kg) showed a significant anxiolytic effect in Ni treated rats as revealed in OF and EPM tests. In addition, exogenous administration of Mel could ameliorate the depressive-like behavior induced by $\mathrm{Ni}$; this neurohormone significantly reduced immobility time in the FST. Consistent with other reports, it was found that Mel produces a variety of anxiolytic-like effects in different tests $[24,37,38]$. Also, Stefanovic et al. showed that Mel elicited a real power antidepressant-like effect in the FST [39]. These findings showed that Mel could prevent anxiety- and depression-like disorders, suggesting a potential neuroprotective role against Ni neurotoxicity. The exact mechanism by which the Mel produces these effects is still under debate.

Another central effect that we highlighted was the effect of $\mathrm{Ni}$ on memory function. Our results also revealed that the chronic administration of Ni causes learning and memory deficits in the MWM test, confirming our previous findings [13]. These results were parallel to a previous report on the association of Ni exposure with memory impairment assessed in the MWM [10]. In the study of He et al., in Mice, acute intoxication with $\mathrm{Ni}$ at 5 and $50 \mathrm{mg} / \mathrm{kg}$ increased the escape latency in the MWM [9]. Significantly, the administration of Mel to Ni-treated rats corrected memory impairment observed in this study, suggesting that Mel exerts a protective effect on Ni-induced cognitive impairment in rats. Further studies will need to investigate the neurobiological mechanism through which Mel modulates cognitive changes.

Accruing data revealed a link between the etiology of affective and cognitive disorders and OS [11, 12]. Generally, the hippocampus, a structure in the brain that plays an important role in emotion regulation, learning, and memory processes [14, 15], is very vulnerable to OS due to their immense oxygen utilization, deficient antioxidant defense, and high polyunsaturated lipid content [40]. Thus, we can suggest that the induction of hippocampal OS by Ni exposure is partially responsible for the behavioral changes observed. Our previous findings indicated that intraperitoneal administration of $\mathrm{Ni}$ for two months caused OS in the hippocampus, emotional illnesses, and impaired spatial learning and memory in rats of both genders [13]. In this context, our results revealed that $\mathrm{Ni}$ exposure induced a remarkable TBARS level (LPO) increase in rat hippocampus. As known, LPO is a major effect of free radicals; the generation of large amounts of free radicals such as $\mathrm{NO}$, superoxide anions $\left(\mathrm{O}_{2} \cdot\right)$, and hydroxyl radical $(\mathrm{OH})$ can directly alter cellular lipids, which may culminate into neuronal damage, leading subsequently to many degenerative illnesses in the CNS [41]. Therefore, the observed elevation of hippocampal LPO in rats treated with $\mathrm{Ni}$ in the current work can be attributed to an overproduction of NO. Ni increased NO formation, which is consistent with our current result [13]. Also, Guan et al. demonstrated that the generation of NO was stimulated by $\mathrm{Ni}$ [42]. At high levels, $\mathrm{NO}$ reacts with $\mathrm{O}_{2}^{-}$to form the ion peroxynitrite (ONOO-), a very reactive toxic molecule that acts as a powerful oxidant of some macromolecules such as lipids, ending by LPO.

By removing free radicals, SOD, and CAT enzymes protect the cells from oxidative damage and prevent LPO [43]. We found herein that the increased LPO and NO levels induced by Ni were accompanied by a significant decrease in SOD and CAT activities in rat's hippocampus. The decreased activities observed may result from the inactivation of the SOD and CAT enzymes due to Ni's direct binding with sulfhydryl groups of enzymes and oxidative modifications of amino acid chains, which alters the protein structure [44].

Mel's capability attenuated the Ni-induced OS might contribute to its protective effects against Ni's neurotoxicity. In this study, we found that Mel administration reduced OS in the hippocampus of both sexes, which was reflected in a decreased level of LPO and NO in Ni treated groups. It has been suggested that Mel's attenuating effect on brain LPO production was mediated by NO neutralization [45]. This neuronal protection may be explained by Mel's alleviation of NO synthase (NOS) enzyme, which down-regulates gene expression of pro-oxidant enzymes, such as NOS [46], and consequently reducing the higher level of NO. In addition, a large body of evidence indicates that Mel and its metabolites can detoxify free radicals and their derivatives such as $\mathrm{ONOO}^{-}$by scavenging both oxygen- and nitrogen-based reactive molecules [47]. Nevertheless, the mechanisms by which Mel balances the NO pathways are still not completely understood. On the other hand, we found that Mel treatment could restore the activity of CAT and SOD enzymes in Ni-treated rats and subsequently prevent the hippocampus neurons from LPO damage. The restoration of these antioxidant enzymes may be due to Mel, which acts as an indirect antioxidant as well by increasing the gene expression and activity of antioxidant enzymes. According to Kotler et al., Mel injections stimulate SOD mRNA levels in rat brains [48]. Collectively, these findings confirmed the protective effect and antioxidant capacity of Mel against oxidative damage.

An increasing body of experimental evidence revealed the pivotal role of OS pathways in neuronal loss [49]. In this study, the biochemical alterations were strongly correlated with the histological analysis. We revealed that Ni exposure provoked a significant neuronal loss in the CA3 subregion of the hippocampus, which plays an essential role in memory and mood function [50]. Therefore, these alterations are associated with behavioral impairments. In contrast, histopathological examination revealed that Mel treatment reduced those hippocampal changes, preserving a normal 
number of pyramidal neurons due to its neuroprotective effect. As discussed above, Mel is protective against neuronal damage in the hippocampus by its potent antioxidant properties, resulting in improved learning and memory [51].

On the other hand, based on our present results, Mel likely can enhance emotional behavior and cognitive capacity by preventing OS in the hippocampus of rats treated with Ni, especially in female rats. El Mrabet et al. (2012) revealed that, in behavior measurement tests, females showed a positive response to Mel treatment compared to males [24]. The difference between the sexes may also be explained by the implication of sexual hormones [52].

\section{Conclusion}

Based on the present study, it can be concluded that chronic Ni treatment caused neurobehavioral and neurochemical changes and a histopathological alteration in rat hippocampus. However, protection against oxidative damage of hippocampus tissues was suggested as a possible mechanism implicated in the beneficial effects of Mel on Niinduced anxiety-like, depression-like and, memory impairment (Figure 6).

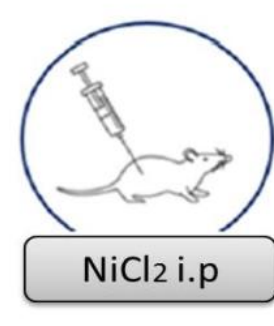

○ Anxiety-like and depression-like behaviors

- Memory impairment Attenuated

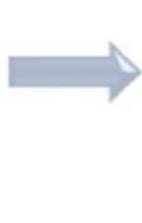

- Oxidative stress and histological changes in the hippocampus

\section{Neuroprotective effect of Melatonin}

\section{Hippocampus}

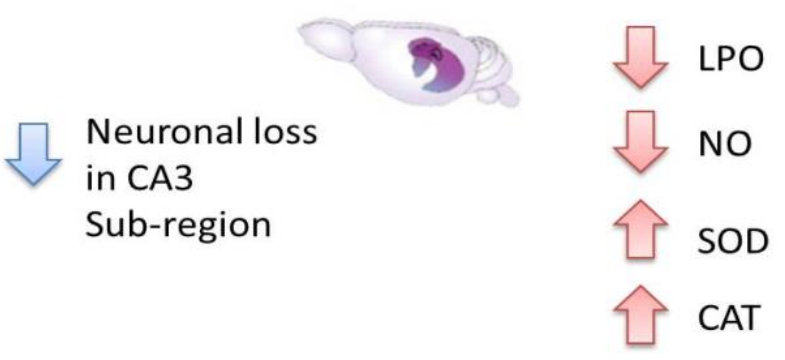

by Melatonin

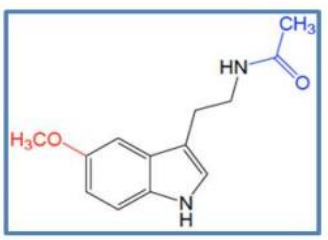

Anxiety-like and

depression-like behaviors

Memory impairment

Figure 6. Overview of Melatonin effects on behavioral, neurochemical and histological parameters in Ni-induced anxiety-like, depressive-like behaviors and memory impairment.

\section{Conflict of interest}

The authors declare no conflict of interest regarding the publication of this manuscript.

\section{CRediT author statement}

ML: Conceptualization, Methodology, Writing-Original draft preparation, Editing; SO, OZ, AEH, HB and SA: Experimentation, Investigation, Visualization, Results compilation; AEH and AM: Data curation and analysis, investigation, Reviewing; AO: Data curation and analysis, Supervision, Reviewing, Editing

\section{ORCID}

Mouloud Lamtai: 0000-0002-4415-7654

Oussama Zghari: 0000-0002-8016-4499

Sofia Azirar: 0000-0002-2564-3630

\section{References}


[1] Das KK, Das SN, Dhundasi SA. Nickel, its adverse health effects \& oxidative stress. Indian J Med Res 2008;128(4):412425.

[2] Huang J, Cui H, Peng X, Fang J, Zuo Z, Deng J, et al. The association between splenocyte apoptosis and alterations of Bax, Bcl-2 and caspase-3 mRNA expression, and oxidative stress induced by dietary nickel chloride in broilers. Int J Environ Res Public Health 2013;10(12):7310-7326.

[3] Marzec Z. Alimentary chromium, nickel, and selenium intake of adults in Poland estimated by analysis and calculations using the duplicate portion technique. Food/Nahrung 2004;48(1):47-52.

[4] Samal L, Mishra C. Significance of Nickel in Livestock Health and Production. Int J Agro Vet Med Sci 2011;5(3):349-61.

[5] Sunderman FW, Dingle B, Hopfer SM, Swift T. Acute nickel toxicity in electroplating workers who accidently ingested a solution of nickel sulfate and nickel chloride. Am J Ind Med 1988;14(3):257-66.

[6] Costa M, Salnikow K, Sutherland JE, Broday L, Peng W, Zhang Q, et al. The role of oxidative stress in nickel and chromate genotoxicity. Mol Cell Biochem 2002;234-235:265-75.

[7] Ijomone OM, Okori SO, Ijomone OK, Ebokaiwe AP. Sub-acute nickel exposure impairs behavior, alters neuronal microarchitecture, and induces oxidative stress in rats' brain. Drug Chem Toxicol 2018;41(4):377-84.

[8] Åkesson B, Skerfving S. Exposure in welding of high nickel alloy. Int Arch Occup Environ Health 1985;56(2):111-7.

[9] He MD, Xu SC, Zhang X, Wang Y, Xiong JC, Zhang X, et al. Disturbance of aerobic metabolism accompanies neurobehavioral changes induced by nickel in mice. Neurotoxicology 2013;38:9-16.

[10] Kahloula K, Adli DEH, Slimani M, Terras H, Achour S. Effet de l'exposition chronique au nickel sur les fonctions neurocomportementales chez les rats Wistar pendant la période de développement. Toxicol Anal Clin 2014;26(4):186-192. (French)

[11] Maes M, Galecki P, Chang YS, Berk M. A review on the oxidative and nitrosative stress (O\&NS) pathways in major depression and their possible contribution to the (neuro)degenerative processes in that illness. Prog NeuroPsychopharmacology Biol Psychiatry 2011;35(3):676-692.

[12] Rahman MF, Wang J, Patterson TA, Saini UT, Robinson BL, Newport GD, et al. Expression of genes related to oxidative stress in the mouse brain after exposure to silver-25 nanoparticles. Toxicol Lett 2009;187(1):15-21.

[13] Lamtai M, Chaibat J, Ouakki S, Zghari O, Mesfioui A, El Hessni A, et al. Effect of Chronic Administration of Nickel on Affective and Cognitive Behavior in Male and Female Rats: Possible Implication of Oxidative Stress Pathway. Brain Sci 2018;8(8):141.

[14] Jangra A, Lukhi MM, Sulakhiya K, Baruah CC, Lahkar M. Protective effect of mangiferin against lipopolysaccharideinduced depressive and anxiety-like behaviour in mice. Eur J Pharmacol 2014;740:337-345.

[15] Sulakhiya K, Kumar P, Jangra A, Dwivedi S, Hazarika NK, Baruah CC, et al. Honokiol abrogates lipopolysaccharideinduced depressive like behavior by impeding neuroinflammation and oxido-nitrosative stress in mice. Eur J Pharmacol 2014;744:124-131.

[16] Lamtai M, Chaibat J, Ouakki S, Berkiks I, Rifi E, El Hessni A, et al. Effect of Chronic Administration of Cadmium on Anxiety-Like, Depression-Like and Memory Deficits in Male and Female Rats: Possible Involvement of Oxidative Stress Mechanism. J Behav Brain Sci 2018;8(5):240-268.

[17] Lamtai M, Ouakki S, Zghari O, Mesfioui A, El Hessni A, Ouichou A. Affective Behavior Dysregulation Was Induced by Chronic Administration of Copper in Wistar Rats. Neurosci Med 2019;10(2):134-149.

[18] Lamtai M, Zghari O, Ouakki S, Marmouzi I, Mesfioui A, El Hessni A, et al. Chronic copper exposure leads to hippocampus oxidative stress and impaired learning and memory in male and female rats. Toxicol Res 2020;36:359_ 366.

[19] Zghari O, Rezqaoui A, Ouakki S, Lamtai M, Chaibat J, Mesfioui A, et al. Effect of Chronic Aluminum Administration on Affective and Cognitive Behavior in Male and Female Rats. J Behav Brain Sci 2018;8(4):179-196. 
[20] Santos ÍMS, Da Rocha Tomé A, Saldanha GB, Ferreira PMP, Militão GCG, De Freitas RM. Oxidative stress in the hippocampus during experimental seizures can be ameliorated with the antioxidant ascorbic acid. Oxid Med Cell Longev 2009;2(4):214-221.

[21] Rehman SU, Ikram M, Ullah N, Alam SI, Park HY, Badshah H, et al. Neurological Enhancement Effects of Melatonin against Brain Injury-Induced Oxidative Stress, Neuroinflammation, and Neurodegeneration via AMPK/CREB Signaling. Cells 2019;8(7):760.

[22] Rodriguez C, Mayo JC, Sainz RM, Antolín I, Herrera F, Martín V, et al. Regulation of antioxidant enzymes: A significant role for melatonin. J Pineal Res 2004;36(1):1-9.

[23] Ouakki S, El Mrabet FZ, El Hessni A, Mesfioui A, Pévet P, Ouichou A. Conversion of L-Tryptophan into Melatonin Is the Possible Action Pathway Involved in the Effect of L-Tryptophan on Antidepressant-Related Behavior in Female Rats: Analysis of the Influence of Treatment Duration. J Behav Brain Sci 2013;3(4):362-372.

[24] El Mrabet FZ, Lagbouri I, Mesfioui A, El Hessni A, Ouichou A. The Influence of Gonadectomy on Anxiolytic and Antidepressant Effects of Melatonin in Male and Female Wistar Rats: A Possible Implication of Sex Hormones. Neurosci Med 2012;3(2):162-173.

[25] Zakaria R, Ahmad AH, Othman Z. The potential role of melatonin on memory function: Lessons from rodent studies. Folia Biol (Czech Republic) 2016;62(5):181-187.

[26] El Mrabet FZ, Ouaaki S, Mesfioui A, El Hessni A, Ouichou A. Pinealectomy and Exogenous Melatonin Regulate Anxiety-Like and Depressive-Like Behaviors in Male and Female Wistar Rats. Neurosci Med 2012;3(4):394-403.

[27] Carola V, D'Olimpio F, Brunamonti E, Mangia F, Renzi P. Evaluation of the elevated plus-maze and open-field tests for the assessment of anxiety-related behaviour in inbred mice. Behav Brain Res 2002;134(1-2):49-57.

[28] Naranjo-Rodriguez EB, Osornio AO, Hernandez-Avitia E, Mendoza-Fernandez V, Escobar A. Anxiolytic-like actions of melatonin, 5-metoxytryptophol, 5-hydroxytryptophol and benzodiazepines on a conflict procedure. Prog NeuroPsychopharmacology Biol Psychiatry 2000;24(1):117-129.

[29] Porsolt RD, Anton G, Blavet N, Jalfre M. Behavioural despair in rats: A new model sensitive to antidepressant treatments. Eur J Pharmacol 1978;47(4):379-391.

[30] Morris R. Developments of a water-maze procedure for studying spatial learning in the rat. J Neurosci Method, 1984;11:47-60.

[31] Kaoud H a, Kamel MM, Abdel-Razek a H, Kamel GM, Ahmed K a. Neurobehavioural, neurochemical and neuromorphological effects of cadmium in male rats. J Am Sci 2010;202(6):54-63.

[32] Draper HH, Hadley M. Malondialdehyde determination as index of lipid Peroxidation. Methods Enzymol 1990;186(C):421-431.

[33] Chao CC, Hu S, Molitor TW, Shaskan EG, Peterson PK, Cha0 CC, et al. injury via a nitric oxide mechanism. Activated microglia mediate oxide neuronal cell injury via a nitric mechanism'. J Immunol 1992;149(8):2736-2741.

[34] Beauchamp C, Fridovich I. Superoxide dismutase: Improved assays and an assay applicable to acrylamide gels. Anal Biochem 1971;44(1):276-287.

[35] Aebi H. Catalase in Vitro. Methods Enzymol 1984;105(C):121-126.

[36] Jalili C, Salahshoor MR, Pourmotabbed A, Moradi S, Roshankhah SH, Darehdori AS, et al. The effects of aqueous extract of Boswellia Serrata on hippocampal region CA1 and learning deficit in kindled rats. Res Pharm Sci 2014;9(5):351358.

[37] Lamtai M, Azirar S, Zghari O, Mesfioui A, Ouichou A. Melatonin Ameliorates Cadmium-Induced Affective and Cognitive Impairments and Hippocampal Oxidative Stress in Rat. Biol Trace Elem Res 2020;1-11

[38] Ouakki S, El Mrabet FZ, Lagbouri I, El Hessni A, Mesfioui A, Pévet P, et al. Melatonin and Diazepam Affect AnxietyLike and Depression-Like Behavior in Wistar Rats: Possible Interaction with Central GABA Neurotransmission. J Behav Brain Sci 2013;3(7):522-533. 
[39] Stefanovic B, Spasojevic N, Jovanovic P, Jasnic N, Djordjevic J, Dronjak S. Melatonin mediated antidepressant-like effect in the hippocampus of chronic stress-induced depression rats: Regulating vesicular monoamine transporter 2 and monoamine oxidase A levels. Eur Neuropsychopharmacol 2016;26(10):1629-37.

[40] Taniguti EH, Ferreira YS, Stupp IJV, Fraga-Junior EB, Mendonça CB, Rossi FL, et al. Neuroprotective effect of melatonin against lipopolysaccharide-induced depressive-like behavior in mice. Physiol Behav 2018;188:270-5.

[41] Khanna by, Ranjana KS, Negi R, Pande D, Khanna S, Khanna HD. Markers of Oxidative Stress in Generalized Anxiety Psychiatric Disorder: Therapeutic Implications Markers of Oxidative Stress in Markers of Oxidative Stress in Generalized Anxiety Psychiatric Disorder: Therapeutic Implications. J Stress Physiol Biochem Orig Text J Stress Physiol Biochem 2012;8(2):32-8.

[42] Guan F, Zhang D, Wang X, Chen J. Nitric oxide and bcl-2 mediated the apoptosis induced by nickel(II) in human T hybridoma cells. Toxicol Appl Pharmacol 2007;221(1):86-94.

[43] Gupta S, Sodhi S, Mahajan V. Correlation of antioxidants with lipid peroxidation and lipid profile in patients suffering from coronary artery disease. Expert Opin Ther Targets 2009;13(8):889-94.

[44] Misra M, Rodriguez RE, Kasprzak KS. Nickel induced lipid peroxidation in the rat: correlation with nickel effect on antioxidant defense systems. Toxicology 1990;64(1):1-17.

[45] Al-Olayan EM, El-Khadragy MF, Abdel Moneim AE. The protective properties of melatonin against aluminiuminduced neuronal injury. Int J Exp Pathol 2015;96(3):196-202.

[46] Tan DX, Manchester LC, Terron MP, Flores LJ, Reiter RJ. One molecule, many derivatives: A never-ending interaction of melatonin with reactive oxygen and nitrogen species? J Pineal Res 2007;42(1):28-42.

[47] Allegra M, Reiter RJ, Tan DX, Gentile C, Tesoriere L, Livrea MA. The chemistry of melatonin's interaction with reactive species. J Pineal Res 2003;34(1):1-10.

[48] Kotler M, Rodríguez C, Sáinz RM, Antolín I, Menéndez-Peláez A. Melatonin increases gene expression for antioxidant enzymes in rat brain cortex. J Pineal Res 1998;24(2):83-89.

[49] Halliwell B. Oxidative stress and neurodegeneration: Where are we now? J Neurochem 2006;97(6):1634-1658.

[50] Zhu C, Xu Q, Wang C, Mao Z, Lin N. Evidence that CA3 is Underling the Comorbidity Between Pain and Depression and the Co-curation by Wu-Tou decoction in Neuropathic Pain. Sci Rep 2017;7(1):1-14.

[51] Soleimani E, Goudarzi I, Abrari K, Lashkarbolouki T. Maternal administration of melatonin prevents spatial learning and memory deficits induced by developmental ethanol and lead co-exposure. Physiol Behav 2017;173:200-208.

[52] Brotto LA, Barr AM, Gorzalka BB. Sex differences in forced-swim and open-field test behaviours after chronic administration of melatonin. Eur J Pharmacol 2000;402(1-2):87-93. 